\title{
Guest editorial: special issue on answer set programming and other computing paradigms
}

\author{
Bart Bogaerts $^{1} \cdot$ Esra Erdem $^{2} \cdot$ Amelia Harrison $^{3}$
}

Published online: 1 May 2019

(C) Springer Nature Switzerland AG 2019

\section{Mathematics Subject Classification (2010) 68 N17}

Answer set programming (ASP) is a well-established knowledge representation paradigm in which combinatorial problems can be expressed in an expressive knowledge representation language. Ever since its introduction in the late 1980s, answer set programming has benefited from, and in turn influenced, related computing paradigms.

The prototypical example of this cross-fertilization is probably the introduction of conflict-driven clause learning in satisfiability solving, which has led to a new generation of answer set solvers. The relationship between ASP and other computing paradigms such as constraint satisfaction (e.g., in the form of constraint answer set programming), satisfiability module theories (e.g., in the form of ASP modulo theories), quantified Boolean formulas (e.g., in the form of nested logic programs), and many other computing paradigms are the topic of active research.

Motivated by this, in 2008, the first workshop on answer set programming and other computing paradigms (ASPOCP) was organized. This tradition has been continued and the 12 th edition in this workshop series is soon to be organized. The current special issue was initiated after the 2017 edition of ASPOCP. It contains extended and revised versions of papers presented at ASPOCP 2016 and ASPOCP 2017, as well as an original contribution.

Unsurprisingly, given the large number of "other computing paradigms", the papers found in this special issue are of a very diverse nature.

This special issue contains three papers originally presented at ASPOCP 2016. Cabalar, Pérez, and Pérez introduce an extension of existential graphs to be used as an alternative

Bart Bogaerts

bart.bogaerts@vub.be

Esra Erdem

esraerdem@sabanciuniv.edu

Amelia Harrison

1 Department of Computer Science, Vrije Universiteit Brussel (VUB), Brussels, Belgium

2 Faculty of Engineering and Natural Sciences, Sabanci University, Istanbul, Turkey

3 New York, NY, USA 
diagrammatic notation for Answer Set Programming, in particular, for its logical formalization in terms of Quantified Equilibrium Logic. van der Hallen, Paramonov, Janssens, and Denecker evaluate ASP and other languages and tools for the problem of graph mining. In their evaluation, they focus on the knowledge representation capabilities of the language and the solving efficiency of the tools. Kahl, Leclerc, and Son describe a new (parallel) solver for the epistemic logic programs, an extension of ASP that recently regained a lot of attention.

Our special issue also contains three papers originally presented at ASPOCP 2017. Cabalar, Constantini, De Gasperis, and Formisano define extensions of multi-context systems, particulary designed for dynamic environments. Fichte, Kronegger, and Woltran study parametric complexity of (disjunctive) answer set programming, thereby drawing a detailed map for a multiparametric view on ASP complexity. Balaii, Gelfond, and Zhang describe improvements of the knowledge representation language P-log that allows for both logical and probabilistic reasoning.

Finally, we accepted one original contribution: Constantini, De Gasperis, and Olivieri use answer set programming for an application in digital forensics, and sketch how such a solution can be integrated in a decision support tool for investigators.

We would like to thank everyone who was involved in this special issue and the workshop series on which it builds, particularly all authors and reviewers. We also thank the editorial and publishing staff at Springer for their support, especially Martin Golumbic, editor-inchief of Annals of Mathematics and Artificial Intelligence.

Publisher's note Springer Nature remains neutral with regard to jurisdictional claims in published maps and institutional affiliations. 